\title{
Estudo epidemiológico das fraturas mandibulares em hospital público da cidade de São Paulo
}

\author{
Epidemiologic study of mandible fractures in a public hospital of São Paulo
}

Antonio Augusto Ferreirinha leporace ${ }^{1}$; Walter Paulesini Júnior ${ }^{1}$; Abrão Rapoport, eCBC ${ }^{2}$; Odilon Victor Porto Denardin ${ }^{3}$

RES U M O

\begin{abstract}
Objetivo: Analisar a frequência epidemiológica de fraturas mandibulares correlacionando gênero, faixa etária, fatores etiológicos, localização anatômica, e tipos de traços de fratura. Métodos: Estudo retrospectivo nos prontuários de 883 pacientes portadores de fraturas faciais, atendidos no Pronto Socorro do Hospital Geral de Vila Penteado, pelo Serviço de Cirurgia e Traumatologia Buco Maxilo Facial (São Paulo - Brasil), num período de três anos (janeiro de 2004 a dezembro de 2006). Resultados: Dos 883 pacientes avaliados, 270 apresentaram fraturas mandibulares (30,5\%). O gênero masculino foi o mais acometido (76,7\%) na faixa etária de 20 a 29 anos (33,0\%), o fator etiológico de maior freqüência foi acidente com veículos automotores (35,2\%), o corpo da mandíbula foi a localização anatômica mais atingida $(47,4 \%)$ e os traços únicos prevaleceram $(76,7 \%)$. Conclusão: As fraturas, em sua maioria, foram simples (traço único), localizadas em corpo mandibular, e destacadas no sexo masculino, na faixa etária de 20 a 29 anos, além do que o fator etiológico mais comum foi acidente com veículos automotores.
\end{abstract}

Descritores: Fraturas mandibulares/epidemiologia. Distribuição por sexo. Distribuição por idade. Traumatologia.

\section{INTRODUÇÃO}

Peserat or ser a mandíbula o único osso móvel da face e estar envolvida em funções de fisiologia complexa, devido a sua posição proeminente em relação ao esqueleto facial e desprotegida, é dos ossos da face, a mais predisposta às fraturas ${ }^{1}$

Muitos estudos feitos em diversos países, têm indicado uma grande freqüência de fraturas mandibulares causada por vários fatores que, por sua vez, variam de acordo com a região analisada, a época e as condições sócioeconômicas. Entre os agentes etiológicos mais comuns, temse o aumento do número de agressões físicas e acidentes automobilísticos, e, em nosso meio o trauma por projétil de arma de fogo, vem se tornando cada vez mais freqüente ${ }^{2}$.

Em nosso país o número de fraturas faciais é elevado, mas poucas pesquisas epidemiológicas têm sido realizadas, apontando, portanto, a necessidade de um maior número e estudos para determinarmos perfis desses traumas, formas de prevenção e adequação dos serviços de atendimento ${ }^{3}$.

Sombrios, os números da violência no trânsito são cada vez mais intoleráveis e já são apontados como um dos maiores problemas de saúde pública do mundo. Segundo a Organização Mundial da Saúde (OMS), o trânsito mata mais de um milhão de pessoas, por ano, e deixa entre 20 e 50 milhões de feridos.
O objetivo deste estudo foi avaliar a freqüência das fraturas mandibulares e correcioná-las como gênero, idade, etiologia, localização anatômica e tipos de traços de fratura.

\section{MÉTODOS}

O projeto de pesquisa deste trabalho foi submetido à avaliação do Comitê de Ética em Pesquisa (CEP) do Hospital Geral de Vila Penteado - São Paulo (Brasil), tendo sua aprovação na data de 20/07/2004, sendo arquivado neste CEP sob o número 319/2004.

Foi realizado um levantamento retrospectivo de 883 pacientes portadores de fraturas faciais, entre janeiro de 2004 a dezembro de 2006, sendo que destes 270 apresentavam fraturas mandibulares, todos os pacientes foram atendidos no Pronto Socorro do Hospital Geral de Vila Penteado

Foram considerados e selecionados para este estudo, somente os registros que se apresentavam preenchidos corretamente, com dados pessoais, anamnese, diagnóstico definitivo e descrição do tratamento, num total de 270 casos.

Os dados foram coletados de um livro de registro de pacientes atendidos no Pronto Socorro, pelos Cirurgiões Buco Maxilo Faciais, de onde inicialmente foram retiradas 
informações pessoais como iniciais do nome do paciente, número do prontuário, gênero, idade, queixa principal, história clínica, exame físico, hipótese diagnóstica, exames de imagem e diagnóstico definitivo.

Todos estes dados foram transcritos pelo autor para uma ficha desenvolvida para coleta de dados desta pesquisa.

Os fatores etiológicos foram divididos em acidentes com veículos automotores, ciclísticos, atropelamentos, agressões físicas, ferimentos por projétil de arma de fogo, quedas, trabalhistas e esportivos.

As fraturas de mandíbula também foram classificadas quanto ao tipo de traços, segundo o qual foram denominadas em simples, que são aquelas representadas por apenas um traço, e múltiplas, que apresentam dois ou mais traços de fraturas.

A estatística descritiva foi contemplada com a apresentação de gráficos com a distribuição absoluta e relativa (\%) das freqüências de cada variável. A associação entre as variáveis nominais (faixa etária, etiologia, gênero, localização da fratura e traços) foi obtida pela aplicação do teste não paramétrico do qui-quadrado, complementado pelo teste exato de Fisher nos casos em que a freqüência esperada de observação fosse menor do que cinco.

E2m todas as situações foi considerado o nível de significância de $5 \%(p<0,05)$ para rejeição da hipótese de igualdade entre os grupos estudados. Todas as análises estatísticas utilizaram o software SPSS ${ }^{\circledR}$, versão 13.0 para Windows (SPSS Inc., Chicago - USA).

\section{RESULTADOS}

A distribuição das fraturas mandibulares segundo o gênero mostra que houve um predomínio para o gênero masculino com $76,7 \%$ (Figura 1). Em relação a faixa etária houve maior freqüência para a faixa etária entre 20 a 29 anos com 33,0\%, seguida pela faixa etária de 10 a 19 anos, com 22,1\% (Figura 2).

A figura 3 mostra que os fatores etiológicos de maior freqüência foram os acidentes com veículos automotores com $35,2 \%$, seguidos pelas agressões físicas com 22,6\%. Foram observadas fraturas mandibulares com maior freqüência nas localizações anatômicas da região de corpo da mandíbula com $47,4 \%$, seguida pela região de côndilo, com 30,7\% (Figura 4).

A distribuição das fraturas mandibulares segundo o tipo de traços mostra que houve um predomínio para as fraturas com traço único com 76,7\% (Figura 5).

A tabulação cruzada, de acordo com a faixa etária e o gênero (Tabela 1) demonstra que houve maior freqüência em homens na faixa etária entre 20 a 29 anos (33,8\%). Não foi observada diferença significativa da distribuição de frequências das fraturas mandibulares em relação ao gênero e faixa etária $(p=0,480)$. Também não foi observada associação entre os fatores etiológicos e o gênero, com distribuição mais freqüentes $(37,7 \%)$ de acidentes com veículos automotores, no gênero masculino $(p=0,089)$. (Tabela 2$)$.

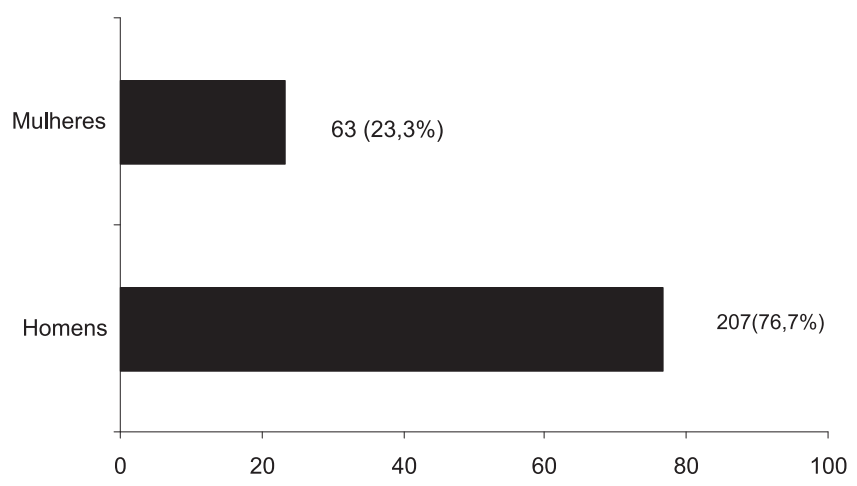

Figura 1 - Distribuição das fraturas mandibulares segundo o gênero.

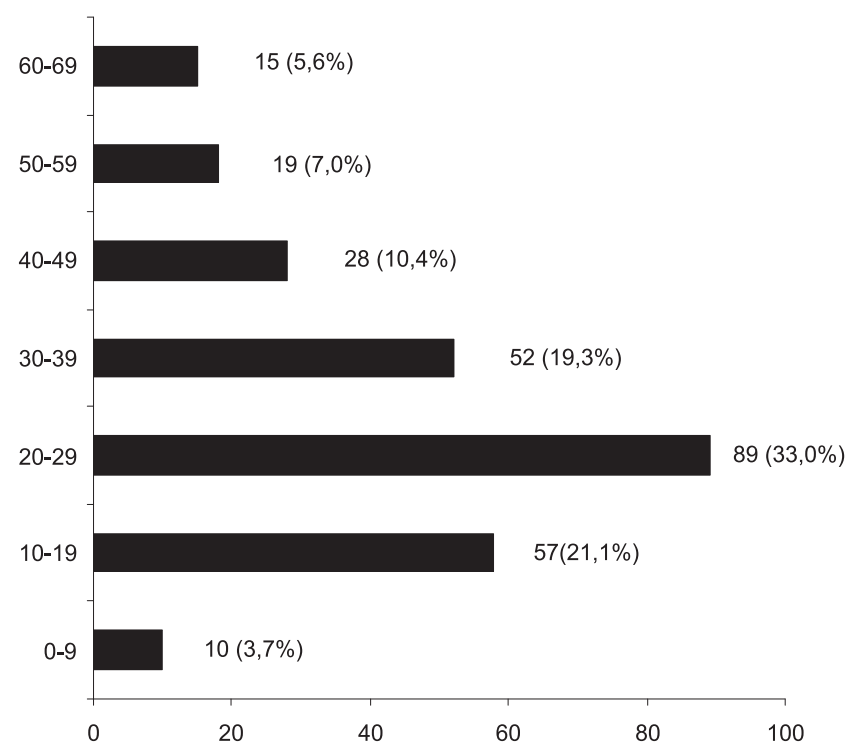

Figura 2 - Distribuição das fraturas mandibulares segundo a faixa etária.

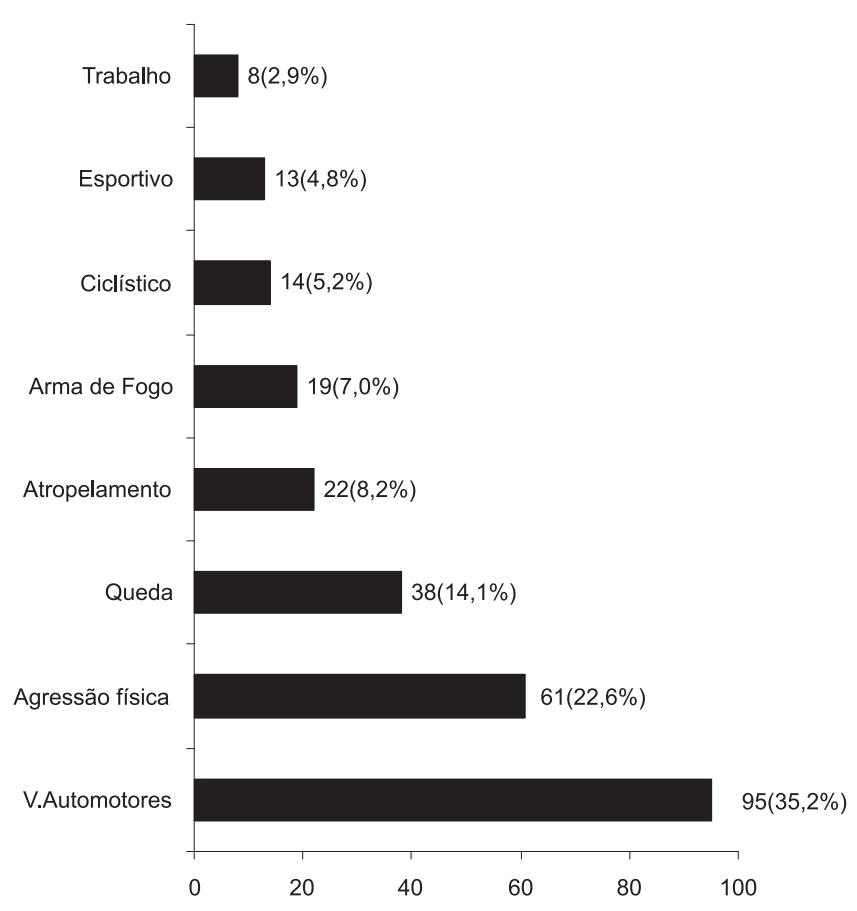

Figura 3 - Distribuição das fraturas mandibulares segundo os fatores etiológicos. 


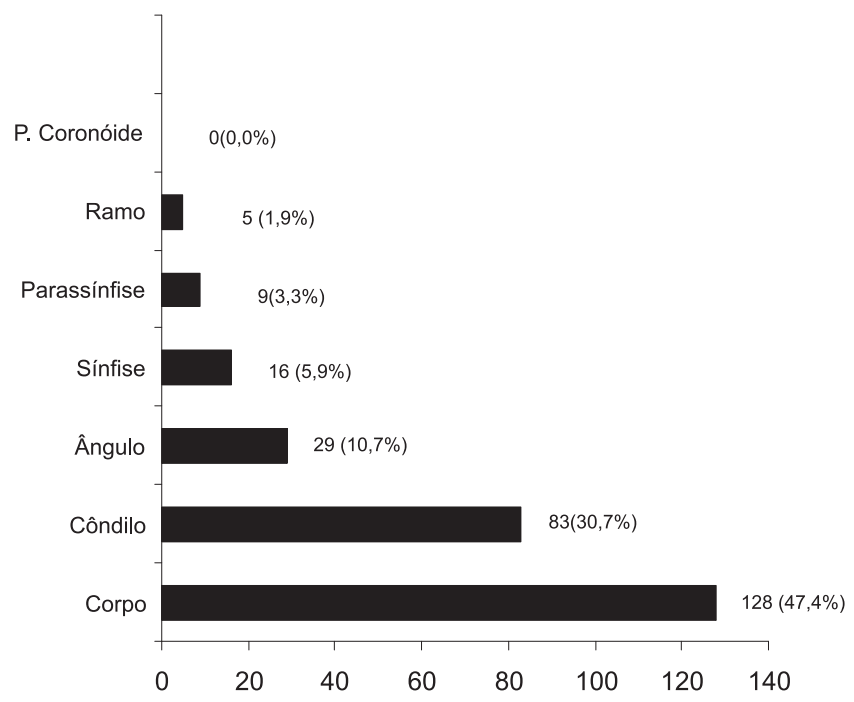

Figura 4 - Distribuição das fraturas mandibulares segundo as localizações anatômicas.

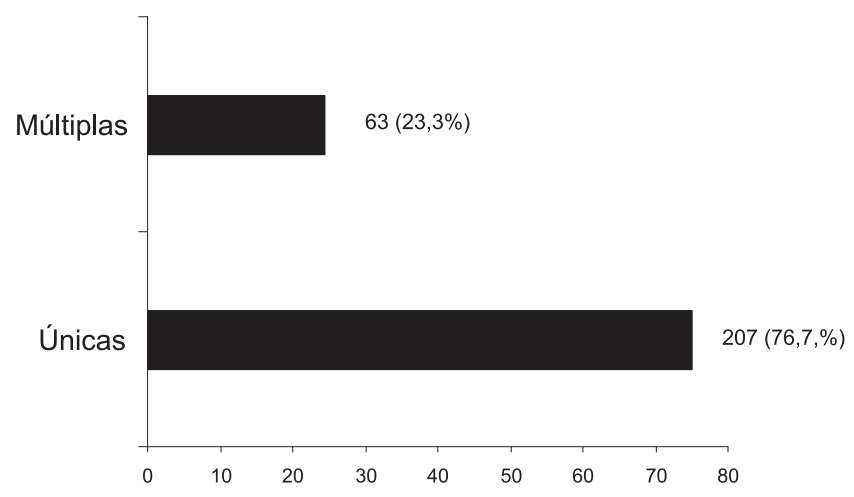

Figura 5 - Distribuição das fraturas mandibulares segundo o tipo de traços.

Quando as fraturas mandibulares foram comparadas, de acordo com a localização anatômica e o gênero, notou-se que houve maior frequência para a região do corpo mandibular no gênero masculino com 51,2\% (Tabela 3), entretanto sem diferença significativa na distribuição entre homens e mulheres $(p=0,105)$.
Tabela 1 - Distribuição dos casos das fraturas mandibulares de acordo com a faixa etária e o gênero.

\begin{tabular}{|c|c|c|c|}
\hline \multirow[t]{2}{*}{ Faixa Etária } & \multicolumn{2}{|c|}{ Gênero } & \multirow[t]{2}{*}{ Total } \\
\hline & Feminino & Masculino & \\
\hline $0-9$ & $(4,8 \%)$ & $(3,4 \%)$ & 10 \\
\hline 10-19 & $9(14,3 \%)$ & $48(23,2 \%)$ & 57 \\
\hline $20-29$ & $19(30,2 \%)$ & $70(33,8 \%)$ & 89 \\
\hline $30-39$ & $14(22,2 \%)$ & $38(18,4 \%)$ & 52 \\
\hline $40-49$ & $7 \quad(11,1 \%)$ & $21(10,1 \%)$ & 28 \\
\hline $50-59$ & $5 \quad(7,9 \%)$ & $14 \quad(6,8 \%)$ & 19 \\
\hline $60-69$ & $6 \quad(9,5 \%)$ & $9 \quad(4,3 \%)$ & 15 \\
\hline Total & $63(23,3 \%)$ & $207(76,7 \%)$ & $270(100 \%)$ \\
\hline
\end{tabular}

A tabela 4 mostra a distribuição das freqüências da faixa etária, de acordo com os fatores etiológicos das fraturas mandibulares. Observou-se uma diferença estatisticamente significativa $(p<0,001)$ nesta distribuição com os acidentes de veículos automotores sendo mais frequente nas faixas etárias de 20 a 29 anos, 30 a 39 anos e 40 a 49 anos, enquanto as faixas etárias menores ( 0 a 9 anos e 10 a 19) tem ocorrência maior de agressão física e acidentes ciclísticos. Nos idosos (mais de 60 anos) observou-se uma freqüência maior de quedas e atropelamentos.

A tabulação cruzada dos casos das fraturas mandibulares de acordo com os fatores etiológicos e o número de traços demonstra que os traços únicos estão associados com maior freqüência as fraturas causadas por acidentes com veículos automotores e agressão física, enquanto os traços múltiplos são associados com arma de fogo e acidentes com veículos. Esta distribuição tem diferença significativa ( $p<0,001$ ) (Tabela 5).

\section{DISCUSSÃO}

O Hospital Geral de Vila situa-se na Região Norte do município de São Paulo, é referência para atendimento nas especialidades em Queimados, Ortopedia e Cirurgia e Traumatologia Buco-Maxilo-Facial não só para a população da região, como também para o Município de São

Tabela 2 - Tabulação cruzada de casos das fraturas mandibulares de acordo com os fatores etiológicos e o gênero.

\begin{tabular}{lrrrrrr}
\hline Fatores Etiológicos & \multicolumn{3}{c}{ Gênero } & \multicolumn{2}{c}{ Total } \\
\cline { 2 - 6 } & \multicolumn{2}{c}{ Feminino } & \multicolumn{2}{c}{ Masculino } & \\
\hline Veículos Automotores & 17 & $(27,0 \%)$ & 12 & $(37,7 \%)$ & 95 \\
Ciclístico & 2 & $(3,2 \%)$ & $(5,8 \%)$ & 14 \\
Atropelamento & 9 & $(14,3 \%)$ & 13 & $(6,3 \%)$ & 22 \\
Agressão física & 16 & $(25,4 \%)$ & 45 & $(21,7 \%)$ & 61 \\
Arma de Fogo & 6 & $(9,5 \%)$ & 13 & $(6,3 \%)$ & 19 \\
Queda & 12 & $(19,0 \%)$ & 26 & $(12,6 \%)$ & 38 \\
Trabalho & 0 & $(0,0 \%)$ & 8 & $(3,9 \%)$ & 8 \\
Esportivo & 1 & $(1,6 \%)$ & 12 & $(5,8 \%)$ & 13 \\
Total & 63 & $(23,3 \%)$ & 207 & $(76,7 \%)$ & $270(100 \%)$ \\
\hline
\end{tabular}


Tabela 3 - Tabulação cruzada das fraturas mandibulares de acordo com a localização anatômica e o gênero.

\begin{tabular}{lcccccr}
\hline Localização Anatômica & \multicolumn{3}{c}{ Gênero } & \multicolumn{2}{c}{ Total } \\
\cline { 2 - 5 } & \multicolumn{2}{c}{ Feminino } & \multicolumn{2}{c}{ Masculino } & \\
\hline Sínfise & 6 & $(9,5 \%)$ & 10 & $(4,8 \%)$ & 16 \\
Coronóide & 0 & $(0,0 \%)$ & 0,0 & $(0 \%)$ & 0 \\
Parassínfise & 2 & $(3,2 \%)$ & 7 & $(3,4 \%)$ & 9 & \\
Corpo & 22 & $(34,9 \%)$ & 106 & $(51,2 \%)$ & 128 & \\
Ângulo & 7 & $(11,1 \%)$ & 22 & $(10,6 \%)$ & 29 & \\
Ramo & 0 & $(0,0 \%)$ & 5 & $(2,4 \%)$ & 5 & \\
Côndilo & 26 & $(41,36 \%)$ & 57 & $(27,5 \%)$ & 83 & \\
Total & 63 & $(23,3 \%)$ & 207 & $(76,7 \%)$ & $270 \quad(100 \%)$ \\
\hline
\end{tabular}

Paulo, por estar localizado próximo às rodovias Via Anhanguera e Bandeirantes, é referência para os casos de acidentes ocasionados nas estradas.

O desenvolvimento industrial do século XX propiciou aumento considerável da frota de veículos em circulação em todo o mundo. Os sistemas viários e o planejamento urbano, em geral, não conseguiram acompanhar o aumento do volume do tráfego. Desta maneira, a qualidade de vida, principalmente nas grandes cidades ficou prejudicada, contribuindo para o aumento da agressividade dos motoristas e para o crescimento da violência no trânsito, o que tem aumentado sensivelmente o número de acidentes com veículos automotores ${ }^{4}$.

Muitos estudos têm indicado uma grande frequência de fraturas mandibulares, que se deve a vários fatores que, por sua vez, variam de acordo com a região analisada, a época e as condições sócio-econômicas. Entre os agentes etiológicos mais comuns, tem-se o aumento do número de agressões físicas e acidentes automobilísticos ${ }^{2}$, e, em nosso meio o trauma por projétil de arma de fogo, vem se tornando cada vez mais freqüente ${ }^{5}$.

Dentre as fraturas faciais, as fraturas de mandíbula foram predominantes ${ }^{1,3,6,7}$. Todavia, para outros ${ }^{5}$, as fraturas nasais são as mais frequentes, seguida pelas fraturas órbito-zigomáticas foram as de maior incidência em fraturas de etiologia por acidente esportivo ${ }^{5}$.

Os achados de nosso estudo coincidem, com os relatos da literatura, em relação a grande maioria das características epidemiológicas das fraturas mandibulares.

Em relação ao gênero, as fraturas mandibulares foram mais frequentes no gênero masculino $0^{3,4,6-10}$.

A incidência de fraturas de mandíbulas é mais acentuada nos homens do que nas mulheres, provavelmente

Tabela 4 - Tabulação cruzada dos casos das fraturas mandibulares de acordo com os fatores etiológicos e a faixa etária.

\begin{tabular}{|c|c|c|c|c|c|c|c|c|}
\hline \multirow[b]{2}{*}{ Fatores Etiológicos } & \multicolumn{7}{|c|}{ Faixa Etária } & \multirow[b]{2}{*}{ Total } \\
\hline & $\begin{array}{c}0-9 \\
\text { anos }\end{array}$ & $\begin{array}{c}10-19 \\
\text { anos }\end{array}$ & $\begin{array}{c}20-29 \\
\text { anos }\end{array}$ & $\begin{array}{c}30-39 \\
\text { anos }\end{array}$ & $\begin{array}{c}40-49 \\
\text { anos }\end{array}$ & $\begin{array}{c}\text { 50-59 } \\
\text { anos }\end{array}$ & $\begin{array}{c}60-69 \\
\text { anos }\end{array}$ & \\
\hline Veículos Automotores & $\begin{array}{c}2 \\
(20,0 \%)\end{array}$ & $\begin{array}{c}6 \\
(10,5 \%)\end{array}$ & $\begin{array}{l}36 \\
(40,4 \%)\end{array}$ & $\begin{array}{l}26 \\
(50,0 \%)\end{array}$ & $\begin{array}{l}17 \\
(60,7 \%)\end{array}$ & $\begin{array}{c}7 \\
(36,8 \%)\end{array}$ & $\begin{array}{c}1 \\
(6,7 \%)\end{array}$ & 95 \\
\hline Ciclístico & $\begin{array}{c}3 \\
(30,0 \%)\end{array}$ & $\begin{array}{c}9 \\
(15,8 \%)\end{array}$ & $\begin{array}{c}1 \\
(1,1 \%)\end{array}$ & $\begin{array}{c}1 \\
(1,9 \%)\end{array}$ & $\begin{array}{l}0 \\
(0,0 \%)\end{array}$ & $\begin{array}{l}0 \\
(0,0 \%)\end{array}$ & $\begin{array}{l}0 \\
(0,0 \%)\end{array}$ & 14 \\
\hline Atropelamento & $\begin{array}{c}2 \\
(20,0 \%)\end{array}$ & $\begin{array}{l}3 \\
(5,3 \%)\end{array}$ & $\begin{array}{l}4 \\
(4,5 \%)\end{array}$ & $\begin{array}{l}2 \\
(3,8 \%)\end{array}$ & $\begin{array}{c}1 \\
(3,6 \%)\end{array}$ & $\begin{array}{c}4 \\
(21,1 \%)\end{array}$ & $\begin{array}{c}6 \\
(40,0 \%)\end{array}$ & 22 \\
\hline Agressão física & $\begin{array}{l}0 \\
(0,0 \%)\end{array}$ & $\begin{array}{l}18 \\
(31,6 \%)\end{array}$ & $\begin{array}{l}31 \\
(34,8 \%)\end{array}$ & $\begin{array}{l}4 \\
(7,7 \%)\end{array}$ & $\begin{array}{c}5 \\
(17,9 \%)\end{array}$ & $\begin{array}{c}2 \\
(10,5 \%)\end{array}$ & $\begin{array}{l}1 \\
(6,7 \%)\end{array}$ & 61 \\
\hline Arma de Fogo & $\begin{array}{l}0 \\
(0,0 \%)\end{array}$ & $\begin{array}{c}6 \\
(10,5 \%)\end{array}$ & $\begin{array}{l}7 \\
(7,9 \%)\end{array}$ & $\begin{array}{l}5 \\
(9,6 \%)\end{array}$ & $\begin{array}{l}1 \\
(3,6 \%)\end{array}$ & $\begin{array}{l}0 \\
(0,0 \%)\end{array}$ & $\begin{array}{l}0 \\
(0,0 \%)\end{array}$ & 19 \\
\hline Queda & $\begin{array}{c}2 \\
(20,0 \%)\end{array}$ & $\begin{array}{l}11 \\
(19,3 \%)\end{array}$ & $\begin{array}{l}6 \\
(6,7 \%)\end{array}$ & $\begin{array}{c}7 \\
(13,5 \%)\end{array}$ & $\begin{array}{l}1 \\
(3,6 \%)\end{array}$ & $\begin{array}{c}4 \\
(21,1 \%)\end{array}$ & $\begin{array}{c}7 \\
(46,7)\end{array}$ & 38 \\
\hline Trabalho & $\begin{array}{l}0 \\
(0,0 \%)\end{array}$ & $\begin{array}{l}0 \\
(0,0 \%)\end{array}$ & $\begin{array}{l}2 \\
(2,2 \%)\end{array}$ & $\begin{array}{l}3 \\
(5,8 \%)\end{array}$ & $\begin{array}{c}1 \\
(3,6 \%)\end{array}$ & $\begin{array}{c}2 \\
(10,5 \%)\end{array}$ & $\begin{array}{l}0 \\
(0,0 \%)\end{array}$ & 8 \\
\hline Esportivo & $\begin{array}{c}1 \\
(10,0 \%)\end{array}$ & $\begin{array}{l}4 \\
(7,0 \%)\end{array}$ & $\begin{array}{l}2 \\
(2,2 \%)\end{array}$ & $\begin{array}{l}4 \\
(7,7 \%)\end{array}$ & $\begin{array}{l}2 \\
(7,1 \%)\end{array}$ & $\begin{array}{l}0 \\
(0,0 \%)\end{array}$ & $\begin{array}{l}0 \\
(0,0 \%)\end{array}$ & 13 \\
\hline Total & 10 & 57 & 89 & 52 & 28 & 19 & 15 & 270 \\
\hline
\end{tabular}


Tabela 5 - Tabulação cruzada dos casos das fraturas mandibulares de acordo com as categorias dos fatores etiológicos e os números de traços.

\begin{tabular}{lrrrrrr}
\hline \multirow{2}{*}{ Fatores Etiológicos } & \multicolumn{3}{c}{ Traços } & \multicolumn{2}{c}{ Múltiplos } & \multirow{2}{*}{ Total } \\
\cline { 2 - 5 } & \multicolumn{2}{c}{ Únicos } & 18 & $(28,6 \%)$ & \multicolumn{2}{c}{95} \\
Veículos Automotores & 77 & $(37,2 \%)$ & 3 & $(3,2 \%)$ & 14 \\
Ciclístico & 11 & $(5,3 \%)$ & 2 & $(3,2 \%)$ & 22 \\
Atropelamento & 20 & $(9,7 \%)$ & 10 & $(15,9 \%)$ & 61 \\
Agressão física & 51 & $(24,6 \%)$ & 17 & $(27,0 \%)$ & 19 \\
Arma de Fogo & 2 & $(1,0 \%)$ & 9 & $(14,3 \%)$ & 38 \\
Queda & 29 & $(14,0 \%)$ & 0 & $(0,0 \%)$ & 8 \\
Trabalho & 8 & $(3,9 \%)$ & 4 & $(6,3 \%)$ & 13 \\
Esportivo & 9 & $(4,3 \%)$ & 63 & $(23,3 \%)$ & $270 \quad(100 \%)$ \\
Total & 207 & $(76,7 \%)$ & & &
\end{tabular}

devido ao maior envolvimento em acidente de tráfego e agressões físicas.

A distribuição pelo gênero parece seguir um padrão na totalidade dos trabalhos pesquisados. Os dados do nosso estudo concordam com os estudos realizados anteriormente, em que a predominância é do gênero masculino $3,6,8,9$.

Em relação à faixa etária, nosso estudo demonstra que a faixa etária de maior ocorrência foi entre 20 a 29 anos, faixa etária esta mais exposta a violência urbana, ratificado por vários estudos ${ }^{2,4,6}$.

Alguns autores utilizaram intervalos que não expressam décadas para apresentação da faixa etária mais comprometida ${ }^{8}$, de 11 a 30 anos; de 16 a 20 anos $^{11}$ e de 16 a 30 anos $^{12}$

Outros autores expressam a ocorrência da faixa etária mais comprometida em médias, que variaram de 27,5 anos até 34,9 anos $^{13}$.

O principal fator etiológico das fraturas mandibulares observado nesta pesquisa foi o acidente com veículos automotores e coincidem com a grande maioria dos trabaIhos pesquisados $1,3,6,7,10,14-16$, seguidos de perto pelas agressões físicas que em outros estudos ${ }^{3,17-19}$ é o principal fator etiológico. O acidente esportivo foi a causa principal de fraturas mandibulares ${ }^{20}$.

Observando-se os dados obtidos de fatores etiológicos nas respectivas faixas etárias, nota-se que os acidentes automobilísticos na faixa de 20 a 29 anos e as agressões e quedas na faixa etária de 30 a 39 anos, foram os índices mais expressivos.

Em relação à localização anatômica, estudos retrospectivos mostraram predominância pela região condilar 12,13,19,21. A região de sínfise e parassínfise foi a mais suscetível às fraturas ${ }^{3}$ seguida pela região de ângulo ${ }^{18,22,23}$. Em nosso estudo, no entanto, demonstramos que a localização mais freqüente de fratura é a região de corpo mandibular, o que concorda com outros estudos $s^{6,14,24,25}$.

Em relação aos tipos de traços, estudos realizados mostram uma maior incidência das fraturas múltiplas $^{13,25}$. Já em nosso estudo houve predominância significativa das fraturas de traço único, concordando com a literatura ${ }^{24,25}$.

O entendimento de todo o processo epidemiológico das fraturas mandibulares mostra que algumas medidas poderiam ser sugeridas para uma diminuição acentuada destes casos, tais como: aplicação adequada das leis de trânsito, controle do consumo de álcool, restrições ao porte de arma de fogo.

Em síntese, concluímos que:

A fratura de mandíbula é mais freqüente em homens, cujas idades variam entre 20-20 anos. O fator etiológico predominante foi o acidente com veículos automotores, sendo mais frequentemente localizada $\mathrm{n}$ corpo da mandíbula, com predomínio de fraturas de traço único.

\section{A B S T R A C T}

Objective: To analyze the frequency epidemiological description of mandibular fractures correlating gender, age, etiological factors, anatomic location, and types of fracture's traces. Methods: A retrospective survey of the medical history of 883 patients with facial fractures, attended at the Buco-Maxilo-Facial Surgery and Traumatology Service of the Hospital Geral de Vila Penteado in São Paulo (São Paulo - Brazil), in a period of 3 years (from January 2004 to December 2006). Results: From 883 patients evaluated, 270 presented mandibular fracture (30,5\%). The male gender was the most affected (76,7\%) in the age of 20 to 29 years $(33,0 \%)$, the most frequent etiological factor was the car accident $(35,2 \%)$, the body of the mandible was the most affected anatomic location $(47,4 \%)$ and the single traces prevailed $(76,7 \%)$. Conclusion: The majority of the fractures were simple, located in the body of the mandible, and higligted in males, aged from 20 to 29 years, and caused by car accident.

Key words: Mandibular fractures/epidemiology. Sex distribution. Age distribution. Traumatology. 


\section{REFERENCIAS}

1. Motamedi $\mathrm{MH}$. An assessment of maxillofacial fractures: a 5-year study of 237 patients. J Oral Maxillofac Surg. 2003; 61(1):61-4

2. Ström C, Johanson G, Nordenram A. Facial injuries due to criminal Violence: a retrospective study of hospital attenders. Med Sci Law. 1992; 32(4):345-53.

3. Andrade Filho EF, Fadul Júnior $R$, Azevedo RAA, Rocha MAD, Santos RA, Toledo SR et al. Fraturas de mandíbula: análise de 166 casos. Rev Assoc Med Bras. 2000; 46(3):272-6.

4. Ellis $E 3^{\text {rd }}$, Moss KF, el-Attar A. Ten years of mandibular fractures: an analysis of 2,137 cases. Oral Surg Oral Med Oral Pathol. 1985; 59(2):120-9.

5. De Gioanni PP, Mazzeo R, Servadio F. La pratica sportive e a traumatologia maxillo-facciale. Attuali aspetti epimiologici e clinici in merito ad una casistica di 379 casi (1982-1998). Minerva Stomatol. 2000; 49(1-2):21-6.

6. Mathog RH, Toma V, Clayman L, Wolf S. Nonunion of the mandible: an analysis of contributing factors. J Oral Maxillofac Surg. 2000; 58(7):746-52.

7. Cabrini Gabrielli MA, Real Gabrielli MF, Marcantonio E, HochuliVieira E. Fixation of mandibular fractures with 2.0-mm miniplates: review of 191 cases. J Oral Maxillofac Surg 2003; 61(4):430-6.

8. Sakr K, Farag IA, Zeitoun IM. Review of 509 mandibular fractures treated at the University Hospital, Alexandria, Egypt. Br J Oral Maxillofac Surg. 2006; 44(2):107-11.

9. Montovani JC, Campos LMP, Gomes MAG, Moraes VRS, Ferreira FD, Nogueira EA. Etiologia e incidência das fraturas de face em crianças e adultos. Rev Bras Otorrinolaringol. 2006; 72(2):235-41.

10. Cheema SA, Amin F. Incidence and causes of maxillofacial skeletal injuries at the Mayo Hospital in Lahore, Pakistan. Br J Oral Maxillofac Surg. 2005; 44(3):232-4. Epub 2005 Jul 27.

11. Klenk G, Kovacs A. Etiology and patterns of facial fractures in the United Arab Emirates. J Craniofac Surg. 2003; 14(1):78-84

12. Ogundare BO, Bonnick A, Bayley N. Pattem of mandibular fractures in an urban major trauma center. J Oral Maxillofac Surg. 2003; 61(6):713-8.

13. David LR, Bisseck M, Defranzo A, Marks M, Molnar J, Argenta LC. Cost-based analysis of the treatment of mandibular fractures in a tertiary care center. J Trauma. 2003; 55(3):514-7.

14. Fischer K, Zhang F, Angel MF, Lineweaver WC. Injuries associated with mandible fractures sustained in motor vehicle collisions. Plast Reconstr Surg. 2001; 108(2):328-31.

15. Boole JR, Holtel M, Amoroso P, Yore M. 5196 mandible fractures among 4381 Active duty army soldiers, 1980 to 1998. Laryngoscope. 2001; 111(10):1691-6.
16. Schön R, Roveda SI, Carter B. Mandibular fractures in Townsville, Australia: incidence, aetiology and treatment using the $2.0 \mathrm{AO} /$ ASIF miniplate system. Br J Oral Maxillofac Surg. 2001; 39(2):145-

17. Sojot AJ, Meisami T, Sandor GK, Clokie CM. The epidemiology of mandibular fractures treated at the Toronto general hospital: a review of 246 cases. J Can Dent Assoc. 2001; 67(11):640-4.

18. Rocton S, Chaine A, Ernenwein D, Rigolet A, Bertrand JC, Ruhin B. [Mandibular fractures: epidemiology, therapeutic management, and complications in a series of 563 cases]. Rev Stomatol Chir Maxillofac 2007; 108(1):3-10; discussion 10-2. Epub 2007 Feb 1.

19. Emshoff R, Schöning H, Röthler $G$, Waldhart E. Trends in the incidence and cause of sport-related mandibular fractures: a retrospective analysis. J Oral Maxillofac Surg. 1997; 55(6):585-92.

20. Dingman RO, Natvig P. Cirurgia das fraturas faciais. $1^{\text {a }}$ ed. São Paulo: Santos; 1983.p.133-90.

21. Asadi SG, Asadi Z. The etiology of mandibular fractures at an urban centre. J R Soc Health. 1997; 117(3):164-7.

22. Wang RC, Keech DR, Elkins TP, Russel P. The tension wire method: a simple, effective means of mandibular fixation. Arch Otolaryngol Head Neck Surg. 1998; 124(4):448-52.

23. Carlin CB, Ruff G, Mansfeld CP, Clinton MS. Facial fractures and related injuries: a ten-year retrospective analysis. J Craniomaxillofac Trauma. 1998; 4(2):44-8

24. Palma VC, Luz JGC, Correia FAS. Freqüência de fraturas faciais em pacientes atendidos num serviço hospitalar. Rev Odontol Univ São Paulo. 1995; 9(2):121-6.

25. Guerrissi JO. Fractures of mandible: is spontaneous healing possible? Why? When? J Craniofac Surg. 2001; 12(2):157-66.

Recebido em 05/12/2008

Aceito para publicação em 11/02/2009

Conflito de interesse: nenhum

Fonte de financiamento: nenhuma

\section{Como citar este artigo:}

Leporace AAF, Paulesini Júnior W, Rapoport A, Denardin OVP. Estudo epidemiológico das fraturas mandibulares em hospital público da cidade de São Paulo. Rev Col Bras Cir. [periódico na Internet] 2009; 36(6). Disponível em URL: http://www.scielo.br/rcbc

\section{Endereço para correspondência:}

Abrão Rapoport

E-mail: arapoport@terra.com.br 